\title{
Comments on "unsuspected femoral hernia in patients with a preoperative diagnosis of recurrent inguinal hernia"
}

\author{
R. Bendavid
}

Received: 29 May 2012/Accepted: 1 July 2012/Published online: 17 July 2012

(C) Springer-Verlag 2012

"To poorly define things is to add to the ills of this world" -Albert Camus.

Femoral hernias, big or small, are the ultimate challenge in hernia surgery. They are relatively uncommon, often overlooked and have traditionally been poorly managed. To surgeons, rookies and veterans alike, they symbolize the baptism of fire. More than for many other surgical problems, they demand an exquisite knowledge of a complex and delicate anatomy. They also demand a close look and analysis of all that is written about them to avoid confusion. Therefore ...

Small femoral hernias may be difficult to diagnose by physical examination and are sometimes identified unexpectedly by laparoscopy.

There is no doubt that the same statement applies to any well-executed approach, laparoscopic or open. Notable exceptions are the onlay mesh techniques and various gadgets since no attempt is made to look at femoral rings from the preperitoneal space.

Laparoscopy sometimes reveals a hernia ... frequency of unsuspected contralateral inguinal hernias is estimated to (sic) 11-28\% in patients planned for unilateral laparoscopic hernia repair ...

"Unsuspected femoral hernia" appears as a leitmotif throughout, but it is never clear whether the aim of the paper is to look at ipsilateral unsuspected (occult, hidden, concealed) hernias only. The author(s) do obtain a

R. Bendavid ( $\square)$

Toronto, ON, Canada

e-mail: rbendavid@sympatico.ca preoperative consent for exploration of the opposite side, in case "a contralateral hernia was clearly visual (sic) without preperitoneal dissection"!

O'Rourke et al. have reported an incidence of $49 \%$ detection by clinical examination as opposed to $71 \%$ laparoscopically $[1,2]$. The more important question is ... whether these hernias which are clinically undetected, asymptomatic, should they be subjected to surgery? Just as importantly, what constitutes an occult hernia? What criteria make an occult hernia an indication for surgery? Asymptomatic hernias have an animistic way of exacting revenge on a surgeon who has broken the practical law that insists that "if it ain't broke, don't fix it"! What surgeon has not seen small, minor, asymptomatic hernias, overzealously treated, develop into giant recurrent hernias?

The preoperative hernia diagnosis was determined by clinical examination or by ultrasonography, if the clinical examination was inconclusive.

The implication is that ALL hernias once detected, by any means, become subject to surgery. That may not sit well with some surgeons.

In this study, we included two patient groups whose preoperative ... bilateral primary inguinal hernias or recurrent inguinal hernia. Patients with a preoperative diagnosis of a femoral hernia or a combination of a recurrent and primary inguinal hernia were excluded.

The bane of all analyses and research ... selection!

Why exclude a patient with the established diagnosis of femoral hernia? There is a contralateral side still which could reveal an "unsuspected hernia" ... the very premise of this article! 
... unilateral primary inguinal hernias were operated on ad modum Lichtenstein and were not included in the study.

A substantial $17.8 \%$ was eliminated ... further selection!

A femoral hernia was defined as tissue protruding through a well-defined hernia ring below the iliopubic tract and medial to the external iliac vein.

The definitions of femoral sheath, femoral ring, femoral orifice and femoral canal left to us by Teale, Cloquet and Fruchaud [3-5] are at variance with the EHS.

What is the structure that forms the constriction and incarceration? Is it the upper femoral ring? Is it the lower femoral orifice? Is it the ring formed by the deeper lamina of the transversalis fascia?

This is the first prospective study to evaluate the frequency of unsuspected femoral hernia in two well defined patient groups.

Some readers may question the choice of the words "first prospective study". It is neither "first" nor "prospective". Previous authors have used terms such as "missed hernias" "occult hernias" or "secondary hernias" instead of "unsuspected hernias" [6-9]. The Shouldice Hospital reports annually and consistently, a 13-14\% incidence of "unsuspected hernias" in combined primary and recurrent hernia operations [8].

The frequency of unsuspected femoral hernias was significantly higher for patients with a preoperative diagnosis of recurrent inguinal hernia.

It is well established that with each subsequent failure of hernia repair, the incidence of re-recurrence becomes progressively higher for all types of hernias especially femoral hernias ... up to $75 \%$ [10-12].

In the present study, $38.1 \%$ of women with a preoperative diagnosis of recurrent inguinal hernias had an occult femoral hernia at surgery compared to $6.6 \%$ in men.

An incidence of $38.1 \%$ is deceiving and must be considered in its proper context. Women rarely get direct inguinal hernias, $0.2 \%$ of all abdominal wall hernias [12]. The majority of their hernias is indirect and, when properly identified and corrected, should never recur, leaving femoral hernias as the only recurrences. In men, where direct and indirect are approximately even in incidence, the majority of recurrences are of the direct variety when surgery has properly eliminated an indirect sac. Is it any wonder women have a higher proportion (not "incidence") of femoral hernias?
Femoral hernias might be overlooked at open groin surgery despite digital exploration of the femoral canal.

This is a spurious claim since a femoral hernia is diagnosed when you identify the femoral ring and the presence of a peritoneal sac being retrieved from that canal. Palpation only, at surgery, would miss a femoral hernia.

Today, laparoscopic repair is widely recommended for women presenting with a hernia in the groin region $[11,12]$.

As for any criteria claimed by a laparoscopist to promote his approach (cost, return to work, pain, recurrence, complications, etc.), the open surgery adept will have an "equal and opposite" substantial, valid counter argument. My personal attitude is pragmatic. Looking at Glassow's large series [13], of all hernias in women, $74.1 \%$ will be of the indirect variety where little need be done beyond resection of the indirect sac and no mesh is needed. As for femoral hernias, mesh is recommended and the choice of approach must be based on the competence of the surgeon in his particular technique.

Another possible explanation for the higher frequency of femoral hernias in patients with recurrent inguinal hernias is that the primary hernia operation might create tension that facilitates the formation of a femoral hernia [11, 12].

So far, we have not seen any detailed breakdowns in the statistics of laparoscopic recurrences, nor convincing histological slides demonstrating pathology. Whereas laparoscopic surgery invariably uses mesh to avoid tension, the results by Neumayer et al. [14] and Huu Le et al. [15] confirm that recurrences following laparoscopy were $10.1 \%$ (Neumayer), and $15 \%$ (Vo Huu Le), for primary hernias, twice as commonly seen for open repair.

A recent study ... concluded that patients who previously underwent Shouldice or Bassini ... and Lichtenstein had severe anatomical changes predisposing to inguinal hernia recurrence and femoral hernia formation.

This is a somewhat self-defeating comment and scenario ... the Lichtenstein repair that has been the mainstay of tension-free repairs has become herniogenic and iatrogenic (!) If this supposition from a pure tissue repair and onlay mesh is true, why would it not be true for an onlay mesh applied laparoscopically?

In conclusion, unsuspected femoral hernias are more prevalent in patients with recurrent inguinal hernias than in patients with primary inguinal hernias. 
I am inclined to think that many surgeons fear to become too aggressive, and for good reasons, with femoral hernias. The femoral vein is a constant reminder of potential danger, especially when dealing with recurrences. Scarring about the femoral triangle can be ligneous and unyielding. The vein can be readily lacerated; it can be constricted by too tight a repair leading to iliofemoral venous thrombosis or invaded by mesh.

Since occult femoral hernias are especially frequent in women with recurrent inguinal hernia, women with a hernia in the groin should always be scheduled for laparoscopic hernia repair.

As stated previously and this needs repeating, a female patient can only have a femoral hernia as a recurrence; my own interest led me to look at a 1981-1984 series covering 28,179 patients who were admitted for surgery $(26,432$ males and 1,747 females). Particularly relevant was the ironclad rule that all posterior inguinal walls were divided to enter the preperitoneal space of Bogros for two reasons (a) the search for femoral and rarer hernias and (b) identifying the best tissues available for repair and to incorporate the lateral edge of the rectus muscle in the repair of the inguinal hernia. A total of 508 femoral hernia repairs were carried out, amounting to $2.17 \%$ of all hernia repairs. The findings are summarized as follows:

There were 251 repairs for primary femoral hernias on 242 patients. There were as well 257 repairs for recurrent femoral hernias on 211 patients. In the group of primary femoral hernia, females accounted for $52.5 \%$. In the group for recurrent femoral hernia, females accounted for $18 \%$ only! While women make up only $6.2 \%$ of all hernias in men and women, it is interesting to observe that in absolute numbers, men and women have the same number of primary femoral hernias. For recurrences, in absolute numbers, men have far more femoral hernias than women 82 versus $18 \%$. What is to be stressed is that as a result, the ratio of primary femoral hernias/primary inguinal hernias is much higher in women, namely $10.13 \%$ while in men, that figure is $1.25 \%$ ! For recurrences, the ratio for women vs men is 2.69 versus $0.79 \%$ [16, 17]. Only the ratios within their own gender do women show a higher reading than men. Neither in absolute numbers nor in relative numbers, do women show a higher incidence of femoral hernias than men!

As a last but perhaps most important criticism, the article appeared, from the very beginning to favor a certain approach, namely laparoscopic surgery, then proceeded to justify it uniquely in that direction. The concern is not so much that the author(s) prefers that technique, everyone is entitled to his/her preference. This criticism comes solidly supported by Professor Schumpelick's address to the
American Hernia Society conference, of February 2005, in San Diego, CA, USA. This memorable address sadly outlined the fact that, "despite the nearly universal use of mesh and the aggressive introduction of laparoscopy, the incidence of hernia recurrences has remained the same in the last 30 years!" [18]. Progress should mean an attempt to solve a Manichean duality into an honest and impartial universality.

By way of a philosophical epilogue, I do want to say to those who pen their thoughts, their research, their discoveries that "clear thinking requires courage, not intelligence" [19].

\section{References}

1. O'Rourke A, Zell JA, Varkey-Zell TT (2002) Laparoscopic diagnosis and repair of asymptomatic bilateral inguinal hernia. Am J Surg 183(1):15-19

2. Cherian PT, Parnell AP (2007) Hernia: CT Findings. Am J Roentgenol 189:W78-W83

3. Teale TP (1846) A practical treatise on abdominal hernia, vol 846. Longman, Green, Brown and Longmans, London, pp 297-337

4. Cloquet J (1817) Recherches Anatomiques sur les Hernies De L'abdomen, vol 1817. Paris, pp 57-75

5. Fruchaud H (2006) The surgical anatomy of hernias of the groin (trans: Bendavid R). University of Toronto Press, pp 130-140

6. Ryan Ernest A (1953) Recurrent hernias. An analysis of 369 consecutive cases of recurrent inguinal and femoral hernias. Surg Gynecol Obstet 96:343-354

7. Welsh DRJ (1975) Inguinal hernia repair. Conn Med 39(2):74-78

8. Bendavid $\mathrm{R}$ The shouldice method of inguinal herniorrhaphy in mastery of surgery. In: Nyhus LM, Baker RJ (eds) 2nd edn, chap 159, table 159-2. Little Brown \& Co, New York, p 1593

9. Obney N, Chan CK (1984) Repair of multiple time recurrent inguinal hernias with reference to common causes of recurrence. Contemp Surg 25:25-30

10. Bendavid R (1995) Femoral hernias: why do they recur? Probl Gen Surg 12(2):147-149

11. Bendavid R (1989) Femoral hernias: primary vs recurrence. Int Surg 74:99-100

12. Bendavid R Mastery of surgery. In: Nyhus, Baker, Fisher (eds) Incision of the floor of the canal, 3rd edn, chap 66. Little Brown \& Co, New York, p 1827

13. Glassow F (1973) An evaluation of the strength of the posterior wall of the inguinal canal in women. Brit J Surg 60(5):342-344

14. Neumayer L, Giobbie-Hurder A, Jonasson O, Fitzgibbons R (2004) Open mesh versus laparoscopic mesh repair of inguinal hernia. NEJM 350:1819-1827

15. Le Huu V, Buffler A, Bertoncello L, Meyer C (2001) Long term recurrence after laparoscopic surgery of inguinal hernias. Hernia $5(2): 88-91$

16. Bendavid R (1989) Femoral hernias: primary versus recurrence. Int Surg 74:99-100

17. Bendavid R (2001) Femoral hernias in females: facts, figures and fallacies. In: Abdominal wall hernias. Springer, pp 639-640

18. Schumpelick V (2005) Address by Pr. Schumpelick at the AHS meeting, San Diego, CA, USA

19. Szasz Thomas Author, professor of psychiatry (b: 1920) 